EPJ Web of Conferences 62, 03003 (2013)

DOI: $10.1051 /$ epjconf/20136203003

(C) Owned by the authors, published by EDP Sciences, 2013

\title{
Nuclear fission in covariant density functional theory
}

\author{
A.V. Afanasjev ${ }^{1, a}$, H. Abusara ${ }^{2}$, and P. Ring ${ }^{3}$ \\ ${ }^{1}$ Department of Physics and Astronomy, Mississippi State University, MS 39762, USA \\ ${ }^{2}$ Department of Physics, Faculty of Science, An-Najah National University, Nablus, Palestine \\ ${ }^{3}$ Fakultät für Physik, Technische Universität München, 85748 Garching, Germany
}

\begin{abstract}
The current status of the application of covariant density functional theory to microscopic description of nuclear fission with main emphasis on superheavy nuclei (SHN) is reviewed. The softness of SHN in the triaxial plane leads to an emergence of several competing fission pathes in the region of the inner fission barrier in some of these nuclei. The outer fission barriers of SHN are considerably affected both by triaxiality and octupole deformation.
\end{abstract}

\section{Introduction}

A study of the fission barriers of nuclei is motivated by their importance for several physical phenomena. For example, the $r$-process of stellar nucleosynthesis depends (among other quantities such as masses and $\beta$-decay rates) on the fission barriers of very neutron-rich nuclei [1]. The population and survival of hyperdeformed states at high spin also depends on the fission barriers [2]. In addition, the physics of fission barriers is intimately connected with on-going search for new superheavy nuclei (SHN). The probability for the formation of a SHN in a heavy-ion-fusion reaction is directly connected to the height of its fission barrier [3]; the large sensitivity of the cross section $\sigma$ for the synthesis of the fissioning nuclei on the barrier height $B_{f}$ also stresses a need for accurate calculations of this value. The survival of the actinides and SHN against spontaneous fission depends on the fission barrier which is a measure of the stability of a nucleus reflected in the spontaneous fission lifetimes of these nuclei [4]. The recent progress in the microscopic description of fission barriers within covariant density functional theory (CDFT) [5] is briefly reviewed in the current manuscript with the main emphasis on SHN.

\section{Comparison with other models}

The progress in the development of computer codes and the availability of powerful computers has allowed to study in a systematic way the effects of triaxiality on the fission barriers leading to their realistic description. Within the CDFT framework, the inner fission barriers with triaxiality included have been studied for the first time in Ref. [6] using the triaxial RMF+BCS approach and the NL3*

\footnotetext{
ae-mail: af ansjev@erc.msstate.edu
}

This is an Open Access article distributed under the terms of the Creative Commons Attribution License 2.0, which permits unrestricted use, distribution, and reproduction in any medium, provided the original work is properly cited. 


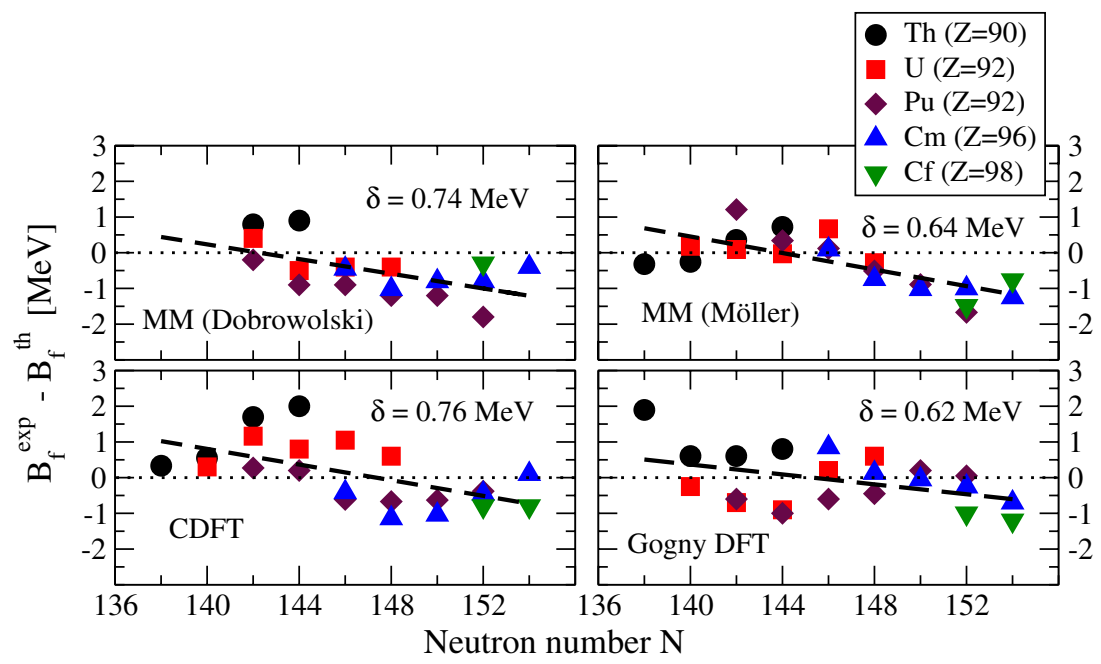

Figure 1. The difference between experimental and calculated heights of inner fission barriers as a function of neutron number $N$. The results of the calculations are compared to estimated fission barrier heights given in the RIPL-3 database [12]. The results of the calculations within the microscopic+macroscopic method ("MM(Dobrowolski)" [13] and "MM(Möller)" [14]), covariant density functional theory ("CDFT" [6]) and density functional theory based on the finite range Gogny D1S force ("Gogny DFT" [15]) are shown. The average deviation per barrier $\delta$ [in MeV] is defined as $\delta=\sum_{i=1}^{N}\left|B_{f}^{i}(t h)-B_{f}^{i}(\exp )\right| / N$, where $N$ is the number of the barriers with known experimental heights, and $B_{f}^{i}(t h)\left(B_{f}^{i}(\exp )\right)$ are calculated (experimental) heights of the barriers. Longdashed lines represent the trend of the deviations between theory and experiment as a function of neutron number. From Ref. [16].

parametrization [7]. Two years later, similar studies of fission barriers in actinides have been performed in Refs. [8, 9] using the RMF+BCS framework with the PC-PK1 [10] and DD-PC1 [11] CDFT parametrizations. The accuracy of the description of the heights of inner fission barriers in these calculations is comparable with the one obtained in Ref. [6]. The calculations of Refs. [8,9] also include the results for outer fission barriers where the effects of octupole deformation (and triaxiality [Ref. [8]]) are taken into account. They agree well with experimental data.

The inclusion of triaxiality has drastically improved the accuracy of the description of inner fission barriers in all model calculations (Ref. [16]). The common consensus is that reflection asymmetric (octupole) deformations do not affect inner fission barriers, but have considerable impact on the outer fission barriers in actinides and SHN. Figure $1^{1}$ shows that the state-of-the-art calculations within different theoretical frameworks (including CDFT) are characterized by a comparable accuracy (the $\delta$-values) of the description of inner fission barriers. Good description of inner fission barriers has also been obtained in the microscopic+macroscopic (MM) calculations of Ref. [17]; however, these calculations do not include Th isotopes. Recent Skyrme DFT calculations are also characterized by similar accuracy of the description of inner fission barriers [18]. The energies of fission isomers and outer fission barriers are also described with the accuracy similar to the one of inner fission barriers in the above mentioned model calculations [19]. Minor differences between the approaches in the obtained average deviations per barrier (Fig. 1) are not important considering appreciable uncertainties in the extraction of fission barrier heights from experimental data (see Ref. [20]).

\footnotetext{
${ }^{1}$ Similar figure but as a function of proton number $Z$ is shown in Ref. [16].
} 

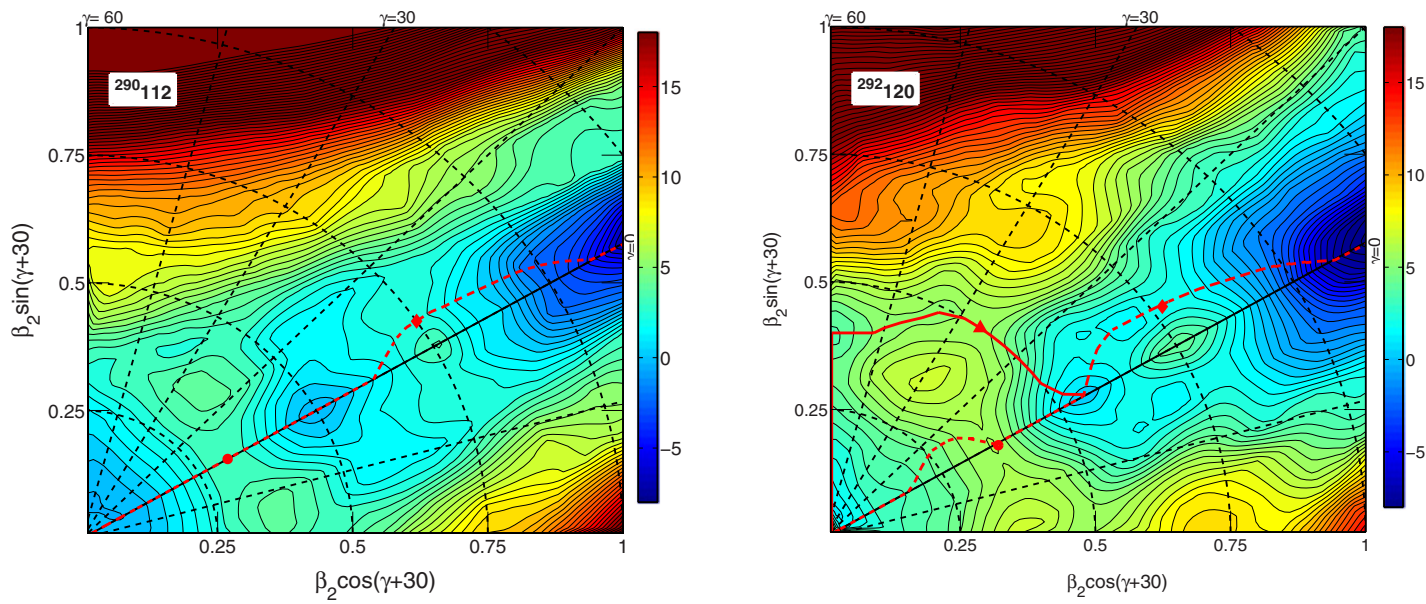

Figure 2. Potential energy surfaces of selected nuclei. The energy difference between two neighboring equipotential lines is equal to $0.5 \mathrm{MeV}$. The saddles along the "Ax" and "Tr-A" fission pathes are shown by solid circles and triangles, respectively. The solid diamonds show the outer fission barrier saddles. From Ref. [16].

The major difference between the models is related to the use of experimental information on the energies of fission barriers or fission isomers in the fit of the model parameters. It turns out that only CDFT studies of Refs. [6, 8, 9] and MM studies of Ref. [13] do not rely on model parametrizations fitted to such data. For example, only spherical nuclei were used in the fit of the NL3* [7] and PC-PK1 [10] CDFT parametrizations which provide a successful extrapolation to fission barriers of actinides [6, 8]. In addition, the DD-PC1 parametrization fitted to the ground states of normal deformed nuclei [11] was used successfully in the CDFT study of fission barriers in Ref. [9]. On the contrary, all successful descriptions of fission barriers in actinides within non-relativistic DFT are based on the parametrizations which explicitly use either fission barrier heights (SkM* [21] in Skyrme DFT and D1S [22] in Gogny DFT) or fission isomer energies (UNEDF1 [18] in Skyrme DFT), which are strongly correlated with the inner fission barrier heights, in the fit of model parameters. It is also necessary to recognize that experimental information on fission barriers have been used in the fit of the macroscopic part of the MM models. For example, the macroscopic part of Ref. [23] used in the calculations of Ref. [17] employs $a_{s}$ and $\kappa_{s}$ parameters of the surface term adjusted to the large heights of experimental fission barriers of relatively light nuclei in Ref. [24]. The parameters of the finite-range liquid-drop model (FRLDM), used in the extensive MM studies of Ref. [14], have also been very carefully fitted to experimental data on fission barriers across the nuclear chart in Ref. [25].

\section{Extrapolation to superheavy nuclei}

Figure 2 shows that the topology of potential energy surfaces (PES) of superheavy nuclei is much more complex than the one in actinides (see Fig. 6 in Ref. [6]) and that it varies drastically with the change of particle numbers. The gross structure of these PES's is defined by the fact that the total energy is generally increasing when moving away from the $\gamma=0^{\circ}$ axis; so it looks like a canyon. However, there are local structures inside the canyon (such as two triaxial hills with moderate deformations $\left(\beta_{2} \sim 0.35, \gamma \sim \pm 30^{\circ}\right)$ which define the variety of fission pathes in the region of inner fission barrier. The axially symmetric fission path (labelled as "Ax") between the normaldeformed/spherical minimum and the superdeformed minimum at $\beta_{2} \sim 0.5-0.6$ is visible in the ${ }^{290} 112$ nucleus. As seen in the ${ }^{292} 120$ nucleus, the $\gamma$-softness of the PES can lead to the development of 


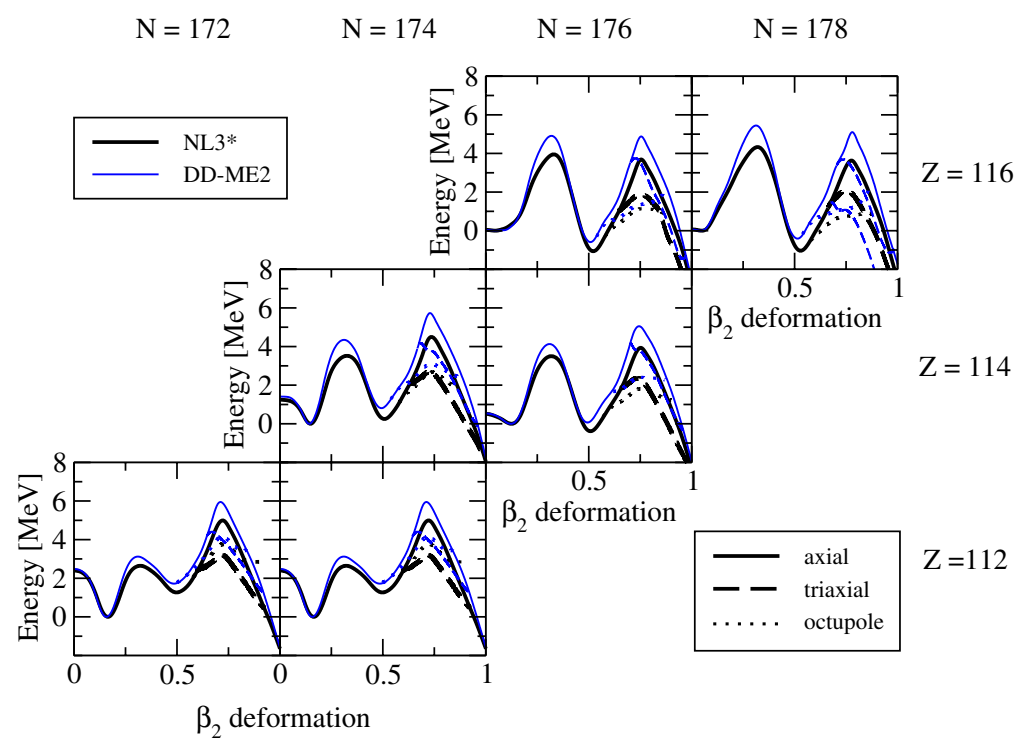

Figure 3. Deformation energy curves for the $Z=112,114$ and 116 nuclei obtained with the NL3* and DD-ME2 parameterizations. Solid lines correspond to axial solutions with reflection symmetry (A), dashed lines to triaxial solutions with reflection symmetry (T), and dotted lines to octupole deformed solutions with axial symmetry (O). Note that the $\mathrm{T}$ and $\mathrm{O}$ solutions are shown only in the deformation range in which they are lower in energy than the axial solution. From Ref. [16].

$\gamma$-deformation along the shoulder of the inner fission barrier without affecting the saddle point. However, in some nuclei this $\gamma$-softness of the PES leads to moderate $\gamma$-deformations $\left(\gamma \sim 10^{\circ}\right)$ at the saddle; then the fission path is called "Ax-Tr". When two triaxial hills with $\left(\beta_{2} \sim 0.35, \gamma \sim \pm 30^{\circ}\right)$ separate from the walls of the PES canyon, a valley between hills and the walls is formed along which the fission path "Tr-A" proceeds. The lowest in energy saddles of these fission pathes are shown below in Figure 4.

The presence of a doubly-humped fission barrier structure in SHN seen in Figure 3 is an example of the most striking difference between the relativistic and non-relativitis calculations; no outer fission barrier appears in absolute majority of non-relativistic calculations in the $Z \geq 110 \mathrm{SHN}$. One can see that the inclusion of triaxiality or octupole deformation always lowers (by around $2 \mathrm{MeV}$ in the majority of the nuclei) the outer fission barrier. The underlying shell structure clearly defines which of the saddle points (triaxial or octupole deformed) is lower in energy. For example, the lowest saddle point is obtained in triaxial calculations in proton-rich nuclei with $N<174$ (Ref. [16]). On the contrary, the lowest saddle point is obtained in octupole deformed calculations in neutron-rich nuclei with $N>174$.

Figure 4 shows how the models which have been benchmarked in a systematic way in the actinides (see Fig. 1) extrapolate to the region of superheavy nuclei. Note that the results of the MM calculations of Ref. [17] labeled as "MM(Kowal)" are not shown on Figure 1. However, they describe inner fission barriers of actinides very accurately. One can see that the model predictions vary wildly; the difference in inner fission barrier heights between different models reaches $6 \mathrm{MeV}$ in some nuclei. This is despite the fact that these models describe the inner fission barriers in actinides with a comparable level of accuracy. The more surprising fact is that the prediction of two MM models differ so substantially; in reality the "MM (Kowal)" model predictions are closer to the CDFT ones than to the "MM (Möller)" predictions.

Let us mention a few possible sources for the differences in the predictions of different models. The different location of the "magic" shell gaps in superheavy nuclei in the macroscopic+microscopic 


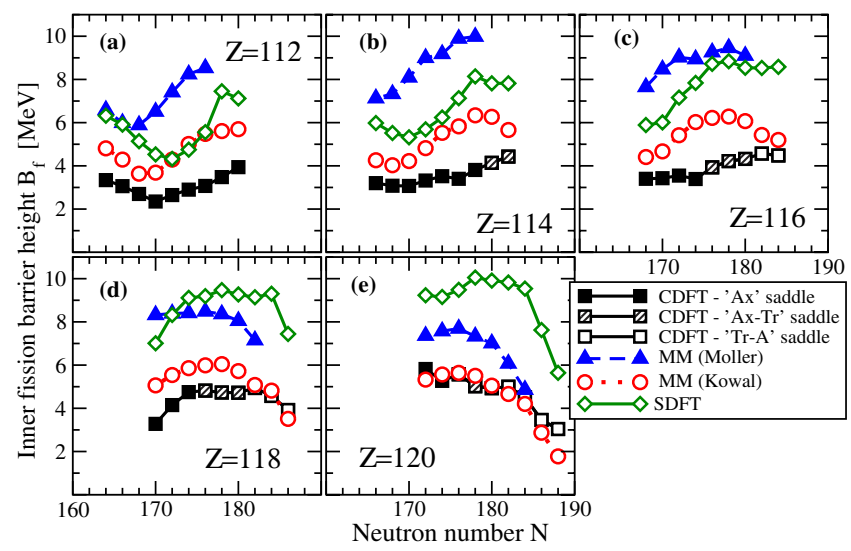

Figure 4. Inner fission barrier heights $B_{f}$ as a function of neutron number $N$. The results of the MM calculations are taken from Ref. [14] (labeled as "MM (Möller)") and Ref. [17] (labeled as "MM (Kowal)"). The results of the Skyrme DFT calculations with the SkM* parametrization (labelled as "SDFT") are taken from Ref. [26]. The position of the inner fission barrier saddle in deformation space varies as a function of particle number. In the case of the CDFT results obtained with the NL3* parametrization, the labeling of Ref. [16] is used in order to indicate whether the saddle is axial (labeled as "Ax"), has small ( $\gamma \sim 10^{\circ}$, labeled as "Ax-Tr") or large ( $\gamma \sim 25^{\circ}$, labeled as "Tr-A") $\gamma$-deformations in the RMF+BCS calculations.

model (at $Z=114, N=184$ ), in Skyrme DFT (predominantly at $Z=126, N=184$ ) and in CDFT (at $Z=120, N=172$ ) results in different single-particle structures at the deformations typical for the ground states and the saddles of the inner fission barriers. These differences will definitely affect the inner fission barriers. The sensitivity of fission barriers to the underlying shell structure can be illustrated by the reduction of the inner fission barrier height of actinides due to triaxiality (Ref. [6]) [or outer fission barrier height due to octupole deformation (Ref. [14])] caused by the level densities in the vicinity of the Fermi surface which are lower at triaxial [octupole] shape as compared with axial one. From our point of view, this represents one of the most dominant sources for the differences in the predictions of the variuos classes of models. However, it does not explain the differences within one class of models seen, for example, in the "MM (Kowal)" and "MM (Möller)" results of Figure 4.

Different pairing schemes are used in the calculations of fission barriers in SHN (see Table IV in Ref. [16] for review). This is another potential source of errors in model calculations, which according to Ref. [27] can reach up to $1 \mathrm{MeV}$. One should note that particle number projection is ignored in the absolute majority of fission barrier calculations. However, the analysis of rotational structures in actinides in Ref. [28] leads to the conclusion that approximate particle number projection by means of the Lipkin-Nogami method decrease the theoretical error bar in the description of the moments of inertia as compared with unprojected calculations. A similar situation maybe expected also for fission barriers. The difference in the effective mass of the nucleon at the Fermi surface does not seem to play an important role (see discussion in Ref. [20]. However, the deficiencies in the treatment of rotational and vibrational corrections (see Refs. [29]) may be responsible for some differences between the various model calculations.

\section{Conclusions}

Covariant density functional theory provides a good description of experimental data on fission barriers in actinides. Contrary to the majority of other models, the fit of these relativistic parametrizations does not include experimental data on fission barriers or isomers. Nonetheless good agreement with 
experiment is achieved in this region. This fact enhances the reliability of CDFT. The similarity of the description of fission barriers achieved by different classes of the theoretical models in actinides does not translate into the similarities of the predictions for superheavy nuclei. This represents a major theoretical challenge for the future.

This work has been supported by the U.S. Department of Energy under the grant DE-FG02-07ER41459 and by the DFG cluster of excellence "Origin and Structure of the Universe".

\section{References}

[1] A. Mamdouh, J. M. Pearson, M. Rayet, and F. Tondeur, Nucl. Phys. A679, 337 (2001)

[2] A. V. Afanasjev and H. Abusara, Phys. Rev. C 78, 014315 (2008)

[3] M. G. Itkis, Y. T. Oganessian, and V. I. Zagrebaev, Phys. Rev. C 65, 044602 (2002)

[4] A. Sobiczewski and K. Pomorski, Prog. Part. Nucl. Phys. 58, 292 (2007)

[5] D. Vretenar, A. V. Afanasjev, G. A. Lalazissis and P. Ring, Phys. Rep. 409, 101 (2005)

[6] H. Abusara, A. V. Afanasjev, and P. Ring, Phys. Rev. C 82, 044303 (2010)

[7] G. A. Lalazissis et al, Phys. Lett. B671, 36 (2009)

[8] B.-N. Lu, E.-G. Zhao, and S.-G. Zhou, Phys. Rev. C 85, 011301(R) (2012)

[9] V. Prassa, T. Nikšić, G. A. Lalazissis, and D. Vretenar, Phys. Rev. C 86, 024317 (2012)

[10] P. W. Zhao, Z. P. Li, J. M. Yao, and J. Meng, Phys. Rev. C 82, 054319 (2010)

[11] T. Nikšić, D. Vretenar, and P. Ring, Phys. Rev. C 78, 034318 (2008)

[12] RIPL-3 is located at http://www-nds.iaea.org/ripl2/

[13] J. Dobrowolski, K. Pomorski, and J. Bartel, Phys. Rev. C 75, 024613 (2007)

[14] P. Möller et al, Phys. Rev. C 79, 064304 (2009)

[15] J.-P. Delaroche, M. Girod, H. Goutte, and J. Libert, Nucl. Phys. A771, 103 (2006)

[16] H. Abusara, A. V. Afanasjev and P. Ring, Phys. Rev. C 85, 024314 (2012)

[17] M. Kowal, P. Jachimowicz, and A. Sobiczewski, Phys. Rev. C 82, 014303 (2010)

[18] M. Kortelainen et al Phys. Rev. C 85, 024304 (2012)

[19] J. D. McDonnell, N. Schunck, and W. Nazarewicz, nuclear theory arXiv:1301.7587

[20] A. V. Afanasjev, H. Abusara, and P. Ring, Int. J. Mod. Phys. E21, 1250025 (2012)

[21] J. Bartel, P. Quentin, M. Brack, C. Guet, and H. B. Hakansson, Nucl. Phys. A386, 79 (1982)

[22] J. F. Berger, M. Girod and D. Gogny, Nucl. Phys. A428, 23c (1984)

[23] I. Muntian, Z. Patyk, and A. Sobiczewski, Acta Phys. Pol. B32, 691 (2001)

[24] P. Möller and J. R. Nix, Nucl. Phys. A361, 117 (1981)

[25] P. Möller, A. J. Sierk, and A. Iwamoto, Phys. Rev. Lett. 92, 072501 (2004)

[26] A. Staszczak, A. Baran, and W. Nazarewicz, Phys. Rev. C 87, 024320 (2013)

[27] S. Karatzikos, A. V. Afanasjev, G. A. Lalazissis, and P. Ring, Phys. Lett. B689, 72 (2010)

[28] A. V. Afanasjev and O. Abdurazakov, submitted to Phys. Rev. C

[29] M. Bender, P.-H. Heenen, and P. Bonche, Phys. Rev. C 70, 054304 (2004) 